\title{
Tratamento intra-operatório da fibrilação atrial crônica com ultra-som
}

\author{
Alexandre Visconti BRICK*, Tamer SEIXAS**, Carlos PORTILHO*, Ayrton Klier PERES**, \\ José Joaquim VIEIRA Jr.*, Romeu de MELO NETO*, Joaquim de Melo ARAÚJO*
}

RBCCV 44205-560

\begin{abstract}
Brick A V, Seixas T, Portilho C, Peres A K, Vieira Jr J J, Melo Neto R, Araújo J M - Tratamento intra-operatório da fibrilação atrial crônica com ultra-som. Rev Bras Cir Cardiovasc 2001; 16(4): 337-49.
\end{abstract}

RESUMO: Introdução: A compartimentação atrial intra-operatória foi realizada em 27 pacientes, utilizando ultra-som (US). Esta forma de energia parece ser mais efetiva na criação de linhas de lesão nos átrios, mais profundas e mais uniformes, sem causar carbonização.

Casuística e Métodos: Foram operados, de março de 1999 e junho de 2000, 27 pacientes, com média de idade de 36 anos. Destes, 19 eram mulheres, 23 eram portadores de doença reumática na valva mitral (5 eram casos de reoperação), 2 apresentavam insuficiência mitral por degeneração mixomatosa, 1 era portador de cardiopatia congênita e 1 apresentava fibrilação atrial isolada.

Resultados: Os tempos operatórios foram em média de 166,6 minutos para a operação, 69,2 minutos de circulação extracorpórea, 39,7 minutos de parada cardíaca pelo pinçamento da aorta, e 12,5 minutos e 14 minutos para efetuar as linhas de ablação nos átrios direito e esquerdo, respectivamente. Houve reversão ao ritmo sinusal em 24 pacientes, durante o ato operatório. Em 2 pacientes a reversão não foi obtida e 1 paciente apresentou bloqueio atrioventricular transitório, não havendo uma explicação plausível para os 2 casos de não reversão, pois os 2 pacientes foram submetidos a operação para correção da valvopatia pela primeira vez e o átrio não estava muito dilatado. Foram observadas duas recorrências e dois óbitos ocorreram por insuficiência respiratória e choque cardiogênico, no pós-operatório imediato, sem relação com a técnica. A porcentagem de sucesso foi de $81,4 \%$, por ocasião da alta hospitalar. Todos os pacientes fizeram uso de verapamil ou amiodarona, para remodelação atrial.

Conclusão: O uso do ultra-som, para criar linhas de ablação no átrio, durante a cirurgia cardíaca, é efetivo, modifica e torna mais fácil o procedimento do labirinto, causa menos dano aos tecidos, diminui o tempo de cirurgia e as possibilidades de complicações pós-operatórias.

DESCRITORES: Fibrilação atrial, cirurgia. Arritmia, terapia. Procedimentos cirúrgicos cardíacos, métodos.

\section{INTRODUÇÃO}

A fibrilação atrial crônica (FAC) é a taquiarritmia sustentada de maior ocorrência na prática médica, tendo maior prevalência na faixa dos 60 anos, aumentando cerca de $2 \%$ a cada década (1). Entre as doenças cardíacas, que mais contribuem para sua instalação, destacam-se hipertensão arterial, miocardiopatia dilatada e doenças valvares, como as lesões por doença reumática, que acometem a população mais jovem (segunda e quarta década de vida, em média), sendo as valvas mitral e aórtica as mais envolvidas.

Hoje se reconhece a importância da fibrilação atrial como fator de risco para acidente vascular cerebral (AVC). Foram desenvolvidas e validadas novas abordagens diagnósticas e terapêuticas efetivas, tais como a ablação e a modificação da condução atrioventricular, com uso do marcapasso.

Técnicas de ablação atrial, com base em estudos clínicos e de ciências básicas, que elucidaram

\footnotetext{
Trabalho realizado no Hospital das Forças Armadas, Hospital Santa Luzia e Cardioclínica. Brasília, DF, Brasil.

Apresentado ao $28^{\circ}$ Congresso Nacional de Cirurgia Cardíaca. Ouro Preto, MG, 5 a 7 de abril de 2001.

* Do Hospital das Forças Armadas e Hospital Santa Luzia, Brasília.

** Da Cardioclínica, Brasília.

Endereço para correspondência: Alexandre V. Brick. SHIS, QL 22, Conj. 03, Casa 6, Lago Sul. Brasília, DF. CEP 71650-235. Tel.: (61) $234-1114$. e-mail: brick@stb.org.br
} 
a eletrofisiologia das arritmias, já obtêm índice elevado de sucesso no controle das arritmias atriais. A associação entre eletrofisiologistas e cirurgiões permitiu o desenvolvimento de métodos cirúrgicos para tratamento da fibrilação atrial crônica, principalmente quando associada à doença valvar.

A primeira intervenção para controlar a fibrilação atrial, refratária ao tratamento clínico, com ablação por cateter da junção atrioventricular, usando choque de desfibriladores, com corrente direta, foi descrita em 1982, por GALLAGHER et al. (2). Essa técnica era eficaz para produzir bloqueio completo e irreversível, com necessidade de implante de marcapasso definitivo. Controlava a freqüência, mas não corrigia os problemas associados à perda da contração atrial e o risco de tromboembolismo.

SCHEINMAN et al. (3) desenvolveram tecnologia com cateter, produzindo ablação do nodo atrioventricular, tornando essa abordagem menos invasiva, sem, contudo, reverter os problemas citados.

MORADY et al.(4) e OLGIN et al.(5) desenvolveram técnicas cirúrgicas alternativas, tentando corrigir as conseqüências deletérias da fibrilação atrial.

Em 1980, WILLIANS et al. ${ }^{(6)}$ desenvolveram a técnica de isolamento do átrio esquerdo, que era capaz de confinar a fibrilação atrial a essa câmara, recuperando o ritmo sinusal nas outras câmaras do coração. A vantagem desse procedimento era sua simplicidade, sendo aplicado nos pacientes operados para correção de valvopatia mitral. Todavia, persistia a fibrilação do átrio esquerdo e o risco de tromboembolia sistêmica.

GRAFFIGNA et al. ${ }^{(7)}$ aplicaram a técnica de isolamento do átrio esquerdo, tanto em pacientes com taquicardia atrial ectópica quanto em portadores de valvopatia mitral cursando com fibrilação atrial.

Em 1985, GUIRANDON et al. ${ }^{(8)}$ introduziram um procedimento de isolamento biatrial, conhecido como "operação do corredor", com o objetivo de isolar uma faixa ou corredor de tecido atrial, situada entre os nódulos sinusal e atrioventricular. Considerando as limitações desse procedimento, foram feitos esforços para criar uma técnica baseada nos mecanismos anatômicos e eletrofisiológicos do "flutter" e da fibrilação atrial, com o objetivo de atingir a cura definitiva.

Através de vários estudos experimentais sobre a eletrofisiologia da fibrilação atrial, foi desenvolvido por COX et al. ${ }^{(9)}$ o procedimento do labirinto, Cox I, em 1991, que empregava secção e sutura, mais crioablação e tinha por objetivo corrigir todas as conseqüências deletérias da fibrilação atrial. Desde sua aplicação inicial, vem sendo modificado com o objetivo de melhores resultados, visando corrigir as irregularidades dos batimentos, permitir o sincronismo atrioventricular e reduzir o risco do tromboembolismo.
A operação de COX et al. $(10,11)$, para fibrilação atrial, descrita originalmente para casos crônicos e paroxísticos, tem tido, em nosso meio, emprego freqüente em pacientes com valvopatia mitral. Foi introduzida, em 1992, por JATENE et al. (12), tendo resultados apresentados em 1995 (13).

Modificações técnicas, incluindo a dispensa da crioablação, foram introduzidas, como as de JAZBIK et al. (14) e GREGORI JÚNIOR et al. (15).

BATISTA et al. (16) propuseram, em 1993, para tratar a fibrilação atrial, autotransplante cardíaco com redução da cavidade atrial esquerda, como alternativa à técnica do labirinto.

$\mathrm{Na}$ tentativa de correção da fibrilação atrial crônica GRIMBERG et al. (17) e MARATIA et al. (18) demonstraram que o simples reparo cirúrgico da valva, ou mesmo sua troca, não garante o retorno ao ritmo sinusal. Está demonstrado que, em pacientes com fibrilação atrial crônica e doença valvar, a combinação do reparo da valva com a realização da técnica do labirinto, idealizada por COX et al ${ }^{(19)}$, permite o retorno ao ritmo sinusal. Embora de eficiência comprovada, na "técnica do labirinto", como geralmente empregada, utiliza-se a dissecção, com abertura da parede atrial, seguida de sutura, o que eleva o tempo de circulação extracorpórea (CEC) e aumenta a chance de complicações no pósoperatório. Para superar estes inconvenientes, os autores idealizaram esta nova abordagem, com aplicação do ultra-som, de maneira a formar linhas de lesões, que determinassem a compartimentação dos átrios esquerdo e direito, com menos tempo cirúrgico e de circulação extracorpórea e conseqüente reflexo nas complicações pós-operatórias.

Constitui objetivo desta investigação a descrição e avaliação inicial dos resultados da técnica para tratamento da fibrilação atrial crônica, com ultrasom, para realização de linhas de ablação, visando a compartimentação atrial.

\section{CASUÍSTICA E MÉTODOS}

Foram estudados 27 pacientes portadores de fibrilação atrial crônica, operados no Hospital das Forças Armadas e Hospital Santa Luzia, em Brasília, no período de março de 1999 a junho de 2000 .

Dezenove pacientes eram do sexo feminino e 8 do sexo masculino. A idade variou de 18 a 64 anos, média de 36 anos.

Vinte e três pacientes eram portadores de valvopatia reumática mitral, assim distribuídos: 8 com diagnóstico de dupla lesão mitral; $6 \mathrm{com}$ insuficiência mitral; 4 com estenose mitral; 5 pacientes tinham sido operados anteriormente para correção de estenose mitral. Dois pacientes apresentavam 
Brick A V, Seixas T, Portilho C, Peres A K, Vieira Jr J J, Melo Neto R, Araújo J M - Tratamento intra-operatório da fibrilação atrial crônica com ultra-som. Rev Bras Cir Cardiovasc 2001; 16(4): 337-49.

insuficiência mitral por degeneração mixomatosa; 1 paciente era portador de cardiopatia congênita, acompanhada de fibrilação atrial crônica (canal atrioventricular, forma parcial) e 1 paciente não apresentava doença valvar, com diagnóstico de "fibrilo flutter" paroxístico, já tendo sido submetido a duas tentativas de ablação endocárdica, sem sucesso.

Os pacientes encontravam-se em classe funcional III ou IV da NYHA (New York Heart Association), apresentavam sintomas relacionados à taquicardia e de insuficiência cardíaca congestiva; 8 pacientes apresentaram episódio prévio de edema agudo de pulmão e 2 deles de acidente vascular cerebral, por fenômeno tromboembólico.

Os pacientes foram submetidos a exames: eletrocardiograma (ECG), ecocardiograma (ECO) e cateterismo cardíaco, com a finalidade de avaliar as valvas cardíacas, presença de trombos intracavitários, tamanho do átrio esquerdo, função ventricular, medidas das pressões em câmaras cardíacas e avaliação das artérias coronárias. O ritmo cardíaco foi avaliado com ECG no pré-operatório, durante o ato operatório, na Unidade de Terapia Intensiva (UTI) e na evolução hospitalar, por ECG e Holter de 24 horas.

Os dados dos pacientes e os diagnósticos são mostrados na Tabela 1 e no Gráfico 1, respectivamente.

As operações realizadas, concomitantemente com a ablação transoperatória, foram: substituição da valva mitral por bioprótese de pericárdio bovino, em 22 pacientes; destes, 5 eram reoperação, plastia valvar mitral em 4 pacientes, associada, em 1 caso, com atrioseptoplastia, e em apenas 1 paciente foi realizada somente a ablação, já que não possuía doença valvar associada (Gráfico 2).

Os procedimentos complementares e associados foram a exclusão das aurículas, esquerda e direita, em 25 pacientes, redução da aurícula esquerda, em 20 pacientes e trombectomia em 5 casos (Gráfico 3).

\section{Técnica Operatória para Realização da Ablação}

Para a realização das linhas de ablação transoperatória foi utilizado o ultra-som, com o Bisturi Harmônico - Ultra Cision ${ }^{\circledR}$, marca registrada da Ethicon Endo Surgery, divisão de Johnson \& Johnson Produtos Profissionais Ltda. São Paulo - SP.

O UltraCision ${ }^{\circledR}$ (Figura 1) é um dispositivo ultrasônico projetado para cortar e coagular tecidos numa freqüência de $55.5 \mathrm{kHz}$ (55.500 ciclos por segundo). Ele é composto de um gerador, pedal de controle, peça de mão e diversos tipos de acessórios. $\mathrm{Na}$ peça de mão encontra-se o transdutor ultra-sônico, que é formado por um bloco de cerâmicas (cristais)

\section{TABELA 1}

Dados dos pacientes portadores de fac submetidos a tratamento intra operatório

\begin{tabular}{lc}
\hline Total de pacientes & 27 \\
\hline Idade & 18 a 64 anos (média $=36)$ \\
Sexo & 19 \\
feminino & 8 \\
masculino & 15 meses \\
Data $(03 / 99$ a 06/00) & 5 a 12 dias \\
Permanência Hospitalar & 6,6 dias \\
\hline Média & \\
\hline
\end{tabular}

\section{GRÁFICO 1 \\ DIAGNÓSTICO PRÉ-OPERATÓRIO DOS PACIENTES PORTADORES DE FAC TRATADOS COM ULTRA-SOM INTRA-OPERATÓRIO}

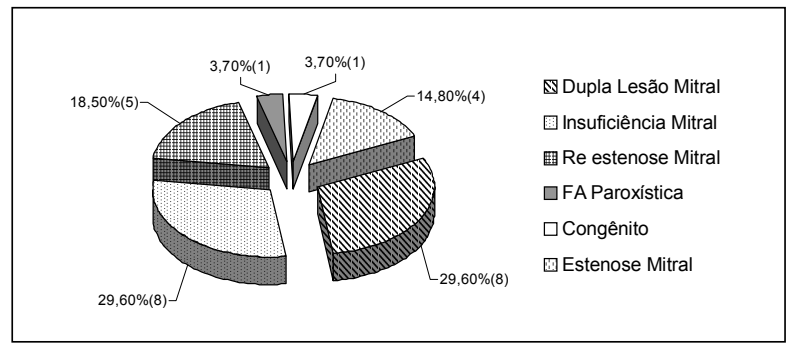

\section{GRÁFICO 2}

PROCEDIMENTOS OPERATÓRIOS EM PACIENTES PORTADORES DE FAC TRATADOS COM ULTRA-SOM INTRA-OPERATÓRIO

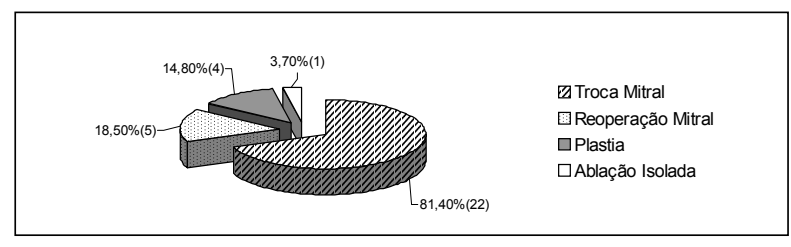

GRÁFICO 3

PROCEDIMENTOS ASSOCIADOS

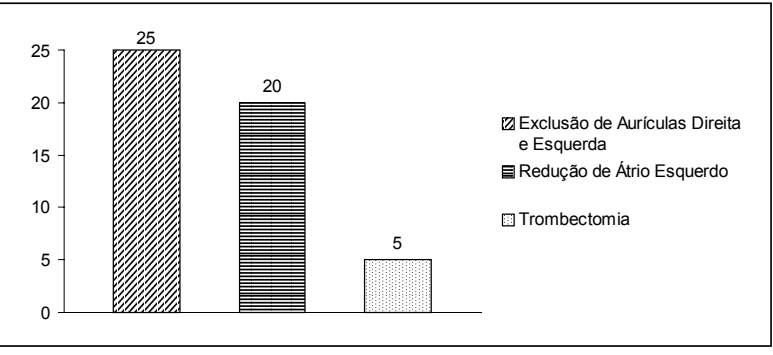

piezo-elétricas prensadas entre dois cilindros metálicos. O transdutor é preso ao amplificador acústico que, por sua vez, é preso ao extensor das hastes, onde se encaixam os instrumentos. 
O gerador é um dispositivo microcompressado, que fornece a corrente elétrica alternada de alta freqüência, para o funcionamento do transdutor na peça de mão. Esta corrente elétrica excita as cerâmicas do transdutor, que vibram na sua freqüência harmônica natural de $55,5 \mathrm{kHz}$. O microcompressador detecta as mudanças no sistema acústico para controlar a energia fornecida à peça de mão e avisa ao usuário as possíveis falhas do sistema. A vibração mecânica do instrumento, através da haste interna do mesmo, que tem o comprimento apropriado a cada tipo de procedimento (Figura 2). A haste interna é protegida por uma camisa externa, cujo diâmetro varia de acordo com a aplicação. Sistema inteiro vibra harmonicamente na freqüência de 55,5 $\mathrm{kHz}$ com um deslocamento máximo de 60 a 100 um $(1 \mathrm{~mm}=0,001 \mathrm{~mm})$.

\section{Procedimentos no Átrio Direito}

No átrio direito foram realizadas ablações lineares no septo interatrial:

1) ao redor da veia cava superior até ao redor da veia cava inferior;

2) da veia cava inferior até a borda inferior do anel da valva tricúspide, passando próximo ao orifício do seio coronário;

3) da veia cava superior até a porção superior do anel tricuspídeo, tomando cuidado com o nódulo atrioventricular (Figura 3).

\section{Procedimentos no Átrio Esquerdo}

Após parada anóxica, pelo pinçamento da aorta, foi efetuada a atriotomia esquerda, longitudinal. As linhas de ablação foram feitas em forma de $U$ invertido, englobando e envolvendo as quatro veias pulmonares, iniciando e terminando no anel fibroso do coração (Figura 4).

Nos pacientes que apresentavam trombos no átrio e aurícula esquerda foi realizada trombectomia, antes do procedimento de ablação, já que a presença dos trombos impedia a confecção das linhas de compartimentação atrial. Após a realização das linhas de ablação, para compartimentação atrial, foram realizados procedimentos de rotina, para tratamento da doença mitral.

O tratamento da doença valvar mitral foi efetuado com troca valvar por bioprótese de pericárdio bovino, pela técnica convencional, plastia valvar e, no paciente portador de cardiopatia congênita, (AVC forma parcial), foi realizada a plastia, com pontos separados e correção do defeito do septo interatrial, com enxerto de pericárdio bovino.

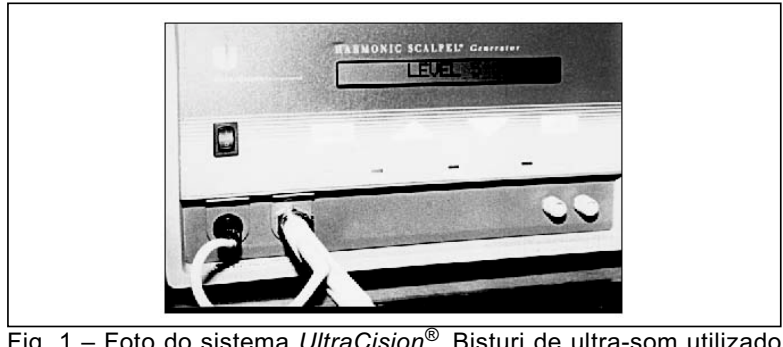

Fig. 1 - Foto do sistema UltraCision ${ }^{\circledR}$. Bisturi de ultra-som utilizado para tratamento intra-operatório da FAC.

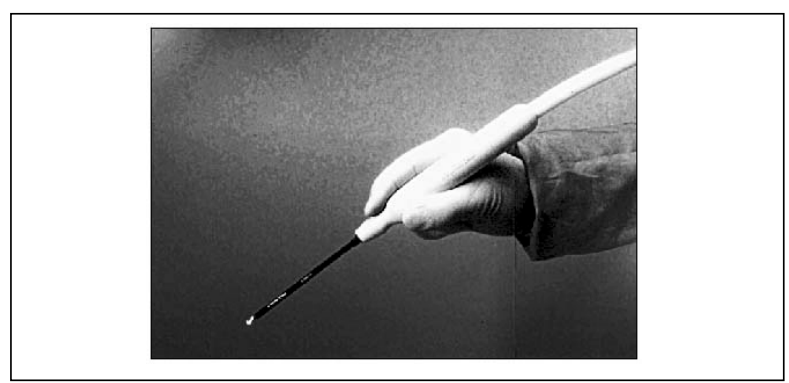

Fig. 2 - Foto do UltraCision ${ }^{\circledR}$ dispositivo ultra-sônico projetado para cortar e coagular tecidos utilizados para tratamento intraoperatório da FAC.

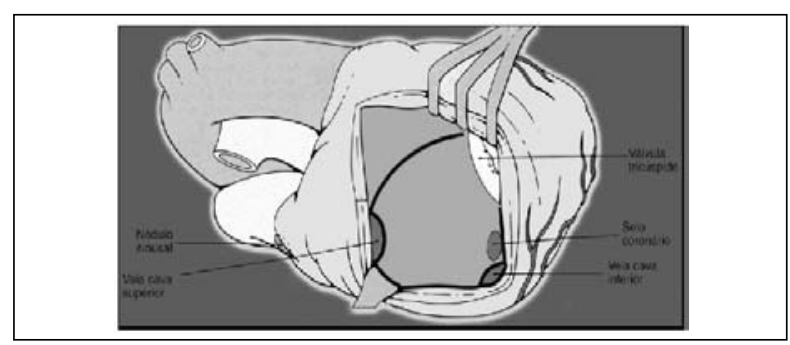

Fig. 3 - Desenho esquemático, mostrando as linhas de ablação no átrio direito, para tratamento intra-operatório da FAC com ultra-som.

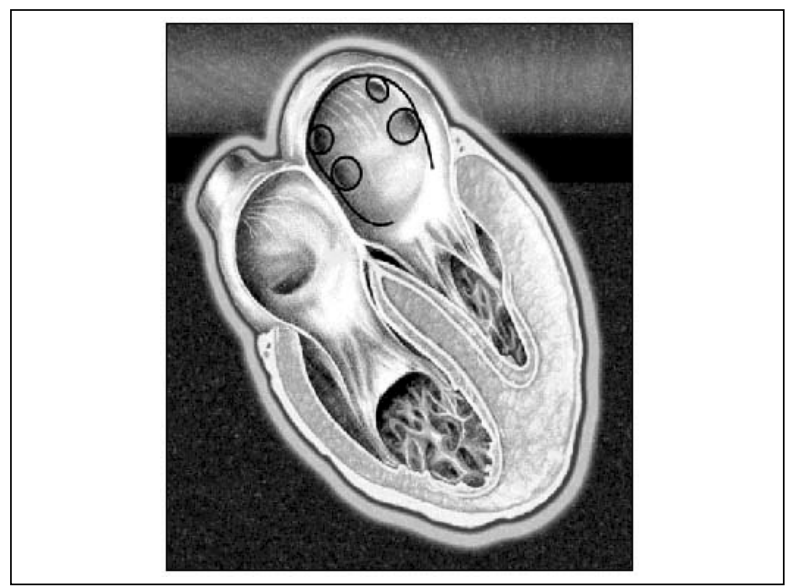

Fig. 4 - Desenho esquemático, mostrando como são realizadas as linhas de ablação no átrio esquerdo, ao redor das veias pulmonares, em forma de $U$ invertido, para tratamento da FAC com ultra-som intra-operatório. 
Brick A V, Seixas T, Portilho C, Peres A K, Vieira Jr J J, Melo Neto R, Araújo J M - Tratamento intra-operatório da fibrilação atrial crônica com ultra-som. Rev Bras Cir Cardiovasc 2001; 16(4): 337-49.

Complementou-se a técnica com exclusão das aurículas direita e esquerda, em 25 pacientes, redução do tamanho do átrio esquerdo, por ressecção e/ou plicatura da parede do átrio esquerdo, em 20 , e trombectomia em 5 pacientes.

Para a estabilização e remodelação atrial, utilizouse verapamil, $120 \mathrm{mg} . / \mathrm{dia}$ e/ou amiodarona, $200 \mathrm{mg} /$ dia, no primeiro dia de pós-operatório. O objetivo foi manter a profilaxia antiarrítmica continuada, devendo permanecer por seis meses nas doses propostas.

\section{RESULTADOS}

Os procedimentos programados foram realizados em todos os pacientes. Após a ablação do átrio direito, com a realização das três linhas já descritas, observou-se modificação da fibrilação atrial, que se mostrou mais organizada.

No átrio esquerdo o procedimento foi realizado com parada anóxica, pelo pinçamento da aorta sem cardioplegia. Após a ablação do átrio esquerdo, observou-se em $24(88,8 \%)$ pacientes reversão ao ritmo sinusal, com contração aparentemente efetiva dos átrios.

Quanto aos resultados intra-operatórios imediatos, $24(88,8 \%)$ pacientes reverteram ao ritmo sinusal. Um paciente apresentou bloqueio atrioventricular total (BAVT), necessitando instalação e estimulação com marcapasso provisório, evoluindo posteriormente para ritmo juncional, não necessitando de implante de marcapasso definitivo e, em 2, não se logrou sucesso durante o procedimento (Gráfico 4).

Quando foram analisados os resultados hospitalares, na ocasião da alta hospitalar, observou-se manutenção do ritmo sinusal em 22 (81,4\%) pacientes, 1 paciente, com BAVT reverteu para ritmo juncional e 2 pacientes apresentaram recidiva da fibrilação no pós-operatório (Gráfico 5).

Ao término do procedimento e final da circulação extracorpórea, todos mantiveram débito adequado, mesmo quando não logramos reverter a fibrilação atrial.

O tempo mediano de operação foi de 166,6 minutos (120-210); o de circulação extracorpórea foi de 69,2 minutos (45-100) e o de pinçamento aórtico foi de 39,7 minutos (20-70).

Os tempos para realização das linhas foram de 12,5 minutos, para o átrio direito e 14 minutos para a realização das linhas no átrio esquerdo.

Nenhum paciente foi reoperado por sangramento.
GRÁFICO 4

TRATAMENTO DA FAC COM ULTRA-SOM RESULTADOS INTRA-OPERATÓRIOS

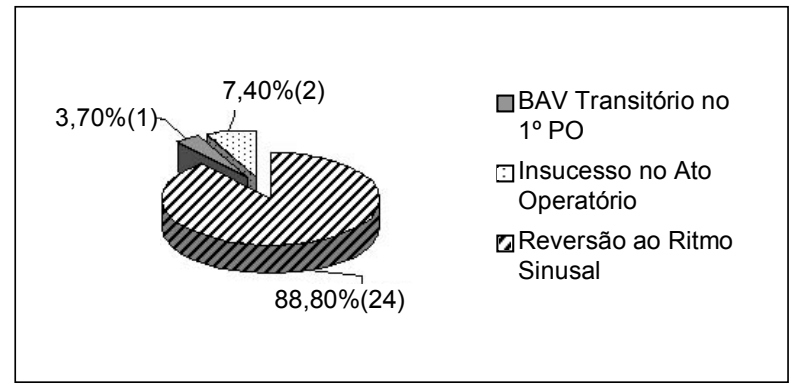

GRÁFICO 5

TRATAMENTO INTRA-OPERATÓRIO DA FAC COM ULTRA-SOM RESULTADOS NA ALTA HOSPITALAR

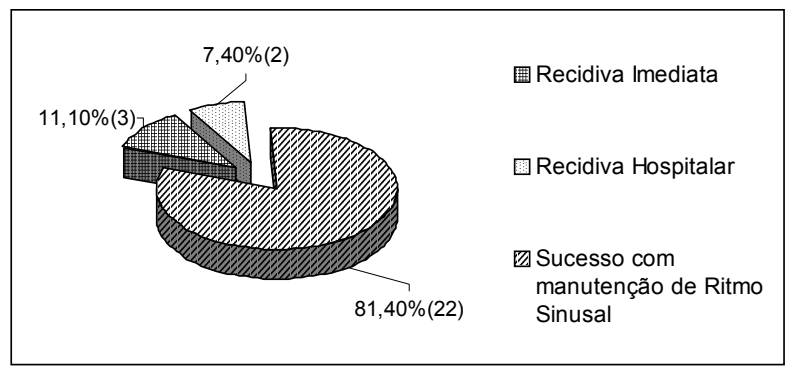

Houve dois óbitos: um paciente de 69 anos, com reestenose mitral calcificada, que apresentou no pósoperatório insuficiência respiratória e pneumonia bilateral; 1 paciente, portador de caquexia mitral, com insuficiência mitral total, grau funcional IV (NYHA), evoluiu com síndrome de baixo débito no pós-operatório imediato, complicando após, com infecção generalizada e falência de múltiplos órgãos.

Em 1 paciente houve rotura da parede do átrio esquerdo, junto das veias pulmonares esquerdas, pelo ultra-som, facilmente corrigida, com sutura de polipropileno $5-0$, pelo interior do átrio. Um paciente apresentou deiscência do esterno, necessitando de revisão da esternotomia, evoluindo bem.

Nenhum paciente necessitou de implante de marcapasso permanente ou desenvolveu bloqueio atrioventricular, no pós-operatório.

Não houve caso de retenção de líquidos, tais como, edema pulmonar, edema periférico ou derrame pleural.

\section{COMENTÁRIOS}

A fibrilação atrial é, dentre as arritmias sustentadas, a de maior prevalência e sua incidência aumenta progressivamente com a idade. É a arritmia supraventricular que mais se relaciona a acidentes 
tromboembólicos e à insuficiência cardíaca e estudos multicêntricos mostram uma incidência de 3 a $7 \%$ ao ano de acidente vascular cerebral, em pacientes que não fazem uso de anticoagulantes (20). O estudo de Framingham (21) mostra um aumento duas vezes maior na mortalidade geral e cardiovascular dos pacientes com fibrilação atrial e MIDDLEKAUF et al. (22) relatam prevalência de 15 a $30 \%$ de FA em pacientes com insuficiência cardíaca.

Atualmente, intensificam-se os estudos da fibrilação atrial, havendo grande interesse em estabelecer novas estratégias no reconhecimento e no tratamento, sejam farmacológicas, intervencionistas, no laboratório de eletrofisiologia ou cirúrgicas. Todavia, apesar de vários métodos farmacológicos e não farmacológicos disponíveis, o impacto do tratamento antiarrítmico na história natural da fibrilação atrial é modesto segundo de PAOLA et al. $(23,24)$.

A fibrilação atrial raramente ocorre antes dos quarenta anos e, nesses casos, há predominância em portadores de doença mitral, reumática ou não, cardiopatias congênitas, síndrome de Wolf-ParkinsonWhite, vias anômalas ocultas, insuficiência cardíaca ou fibrilação isolada. A maior prevalência, em nosso país, é provocada pela valvopatia reumática. A fibrilação atrial está associada às valvopatias reumáticas, principalmente às doenças da valva mitral, com incidência de $43 \%$ nos portadores de insuficiência ou estenose mitral (25). Alguns estudos mostram incidência de até $75 \%$ de fibrilação atrial, em valvopatias reumáticas mitrais (26).

Em passado recente, havia a idéia de que o tratamento isolado da valva mitral poderia restabelecer o ritmo sinusal. Todavia, trabalhos de GRIMBERG et al. (17) e MARATIA et al.(18) mostraram reversão para ritmo sinusal em $37 \%$ e $28 \%$, respectivamente, nos pacientes portadores de fibrilação atrial, após intervenção na valva mitral. A manutenção da fibrilação atrial aumenta o risco de fenômenos tromboembólicos ${ }^{(27)}$, sendo necessário o uso de anticoagulação oral e, já no pós-operatório imediato, existe diferença, principalmente na manutenção do débito cardíaco, em pacientes que não reverteram a fibrilação atrial devido às conseqüências adversas, geradas pela perda da contração dessa câmara. FISHER ${ }^{(28)}$ relata que cerca de $33 \%$ de todos os episódios de tromboembolismo estão relacionados com a fibrilação atrial e destes, $60 \%$ podem produzir seqüela ou morte.

A eletrofisiopatologia da fibrilação atrial não está totalmente elucidada. A teoria mais aceita foi proposta por MOE (29), em 1962, e ALLESSIE et al. (30), em 1985, e sugeria que várias ondas reentrantes e desordenadas propagavam-se pelos átrios.
Diversas ondas ou impulsos de ativação independentes trafegam contínua e aleatoriamente através do miocárdio despolarizável, circundando regiões onde há bloqueio funcional da condução. Esses impulsos têm intensidade variável e chegam ao nódulo atrioventricular de forma irregular, conforme relatam FENELON \& de PAOLA (31).

A condução pelo nódulo atrioventricular é sensível ao tônus autônomo; a redução do estímulo vagal ou o aumento da atividade simpática facilita a condução. A fibrilação atrial pode se perpetuar por alterações estruturais e funcionais dos átrios e está associada à dilatação atrial progressiva.

Um dos melhores preditores para o desenvolvimento da fibrilação atrial é o tamanho dos átrios (32); quanto maior for a quantidade de tecido disponível, mais provável será a formação e a perpetuação de um circuito reentrante; deste modo quanto maiores forem os átrios, mais as ondas de ativação, que constituem a fibrilação atrial, encontram tecido em condições de ser despolarizado, perpetuando a arritmia (33,34). O aumento dos átrios, que acompanha diversas cardiopatias, leva, freqüentemente, ao desenvolvimento de fibrose intersticial e desconexão elétrica entre os miócitos atriais, provocando alentecimento da condução em vários segmentos de tecido, favorecendo a diminuição do comprimento da onda e a indução e perpetuação da fibrilação atrial $(35,36)$.

A fibrilação atrial aumenta sua incidência com o avançar da idade e provoca uma série de problemas, sendo que três são mais importantes: irregularidade, com freqüência cardíaca elevada, levando a quadro de taquimiocardiopatia; comprometimento hemodinâmico, com perda do sincronismo atrioventricular e trombose atrial, com risco de fenômenos tromboembólicos.

Considerando a morbidade e a mortalidade da fibrilação atrial, é perfeitamente válido que alguma técnica ou procedimento seja feito, principalmente durante o tratamento da doença mitral, na tentativa de eliminar a arritmia e restabelecer o ritmo sinusal.

No presente estudo, 25 pacientes eram portadores de doença valvar mitral, sendo 23 reumáticos e 2 provocados por degeneração mixomatosa com conseqüente aumento do tamanho dos átrios. $O$ estudo realizado com ecocardiograma mostrou tamanho médio de átrio esquerdo em torno de $60 \mathrm{~mm}$.

Várias técnicas cirúrgicas têm sido experimentadas para eliminar a fibrilação atrial ou reduzir os riscos de complicações relacionadas à arritmia. Entre as já citadas, encontramos: 1. a técnica do isolamento atrial (6) esquerdo, que consiste num proce- 
dimento relativamente simples, que não prolonga o tempo de circulação extracorpórea, e foi proposta para pacientes submetidos a troca valvar. GRAFFIGNA et al. (7), em 1992, realizaram esta técnica em 100 pacientes, obtendo o ritmo regular em $81,4 \%$ dos casos e, dois anos após, $70 \%$ dos pacientes estavam mantendo o ritmo. Todavia, o átrio esquerdo continuava em fibrilação, não diminuindo o risco de tromboembolismo. 2. a fulguração do feixe de His por cateter e implante de marcapasso definitivo (3) ; 3. a "cirurgia do corredor" (8), que isola as paredes livres dos átrios, criando um corredor no septo interatrial, ligando o nódulo sinusal à junção atrioventricular. Embora esta técnica mantenha o ritmo sinusal, não elimina a fibrilação do átrio esquerdo, não restabelece o transporte atrial e não diminui o risco de tromboembolismo.

Os estudos experimentais, desenvolvidos por BOTTERON E SMITH (37) e COX et al. (38) na última década, usando-se o mapeamento computadorizado intraoperatório, capaz de registrar simultaneamente a ativação elétrica de 160 pontos atriais, permitiram documentar a seqüência da ativação atrial e melhor compreender os fenômenos elétricos durante a fibrilação.

A fibrilação atrial consiste de pequenos e múltiplos circuitos micro reentrantes, localizados no tecido atrial, apresentando intervalos de ciclo pequenos e variáveis, que necessitam de uma massa crítica de tecido contínuo para sua manutenção. Todavia, esses circuitos são transitórios no tempo e no espaço, migrando de um local para outro e desaparecendo.

Esses conhecimentos nortearam a estratégia da cirurgia do labirinto proposta por COX et al. $(39,40)$. A operação de Cox é um procedimento cirúrgico que realiza múltiplas incisões atriais, criando um trajeto definido para o estímulo atrial. Como na "cirurgia do corredor", foi realizado o direcionamento da ativação atrial, do nódulo sinusal até o nódulo atrioventricular, através de linhas cirúrgicas de constrangimento ou compartimentação da condução dos estímulos elétricos atriais. As incisões estendem-se ao restante do átrio direito e todo o átrio esquerdo, fazendo com que a ativação elétrica, que se origina no nódulo sinusal, progrida entre as linhas de sutura, inferiormente e anteriormente, em volta da base do átrio direito para a porção cranial do septo interatrial. A ativação é assim separada em duas frentes de onda, uma ativando as paredes póstero-mediais dos átrios e a outra a parede póstero-lateral do átrio esquerdo, finalmente propagando-se ao nódulo atrioventricular. Este modelo de ativação foi desenhado para preservar o sincronismo atrioventricular.
A operação de Cox (9) foi originalmente descrita para tratamento da fibrilação atrial crônica ou paroxística isoladas. Relatos de COX et al. ${ }^{(41)}$ mostraram que em 75 pacientes operados, 93\% apresentavam fibrilação crônica ou paroxística isoladas. Em nosso meio, a maioria dos relatos da operação de Cox foi realizada em associação com outras doenças cirúrgicas, principalmente à doença valvar, conforme os relatos de experiência inicial de JATENE et al.(12), em 1992 e GREGORI JR. et al. (15), em 1993.

Nos casos de FA associadas com valvopatias, JATENE et al. $(42,43)$ estudaram 55 casos com fibrilação atrial e valvopatia reumática e concluíram que a operação de Cox mostrou ser eficiente no controle da arritmia, devendo ser considerada como opção terapêutica nesses pacientes. Entretanto, todos os autores citados a respeito demonstram ser a operação de Cox procedimento que demanda tempo operatório maior, mesmo quando realizada deforma isolada: SANDOVAL et al. ${ }^{(44)}$ e MACARTHY et al. (45) referiram tempo prolongado de circulação extracorpórea e de parada anóxica. KOSAKAl et al. ${ }^{(46)}$, em pacientes com lesão valvar mitral, demonstraram tempo médio de circulação extracorpórea de 226 minutos e tempo médio de pinçamento aórtico de 142 minutos. KAWAGULIE et al. (47) também mostraram tempos prolongados, analisando os riscos e benefícios do procedimento do labirinto.

Outro fato também citado como limitante da realização da operação de Cox, como demonstram LOZANO et al. (48), é a maior incidência de complicações no pós-operatório, principalmente a tendência de reter líqüidos, levando a edema e congestão pulmonar, maior tempo de intubação e permanência na UTI.

Dentre as complicações são citadas: secção dos átrios, levando insuficiência cardíaca direita e esquerda, maiores tempos de operação e de circulação extracorpórea e permanência na UTI e risco de sangramento, pela grande quantidade de cortes e suturas.

$\mathrm{Na}$ casuística analisada, os tempos operatórios foram em média de 166,6 minutos, o de circulação extracorpórea de 69,2 minutos, para a realização da ablação intra-operatória foi de 13,2 minutos e não registramos complicações relacionadas ao procedimento, exceto uma lesão da aurícula esquerda, junto das veias pulmonares, provocada pelo bisturi de ultra-som, que foi facilmente suturada.

Com o advento das técnicas de ablação transcateter, usando a radiofreqüência, tornou-se possível curar grande parte das arritmias supraventriculares. 
O sucesso dos resultados alcançados na cirurgia do labirinto, que demonstrou a possibilidade de cura da fibrilação atrial, motivou um grande interesse na reprodução da técnica do labirinto por meio da ablação por cateter.

Estudos iniciais em laboratório realizados por AVITALL et al. (49) e ELVAN et al. (50) demonstraram a possibilidade de se fazer lesões lineares de radiofreqüência, em átrios de cães, impedindo, assim, a reindução da fibrilação atrial.

Os trabalhos de ablação transcateter em laboratórios de eletrofisiologia, realizados por SWARTZ et al. (51), reproduziram a técnica do labirinto. Demonstraram ser possível a cura da fibrilação atrial, pela ablação com radiofreqüência, usando bainhas vasculares desenhadas, com curvas pré determinadas, reproduzindo a técnica do labirinto, por meio de oito lesões lineares, três no átrio direito, quatro no átrio esquerdo e uma no septo interatrial.

Em 1994, HAISSAGUERRE et al. (52) descreveram o primeiro caso de ablação com cateter para tratamento da fibrilação atrial. Com um cateter especial, foram realizadas lesões lineares no átrio direito: a primeira vertical na parede posterior, a segunda estendendo-se da região ântero-lateral média do átrio direito à região póstero-septal do anel tricuspídeo, no plano horizontal e a terceira, na região anterior, conectando os pontos terminais das duas primeiras.

Posteriormente, o mesmo grupo ${ }^{(53)}$ relatou uma série de ablações com radiofreqüência. Foram realizadas linhas no átrio direito em todos os pacientes, e, em alguns, também no átrio esquerdo.

Do mesmo modo, observaram que, na maioria dos casos, a ablação isolada do átrio direito apenas regularizava a atividade elétrica, enquanto que a ablação do átrio esquerdo, isolada ou após ablação do átrio direito, era eficaz para restaurar o ritmo sinusal. As limitações do procedimento são ao tempo prolongado de sala e emissão de Raio-X, o risco de tromboembolismo e a estenose das veias pulmonares (54).

Apesar dos resultados descritos por GAITA et al. (55) e GARG et al. (56), a ablação da FA, com cateter, baseada na cirurgia do labirinto, ainda está em fase experimental.

A criação de lesões lineares contínuas é um dos maiores desafios, considerando-se todas as irregularidades da anatomia atrial, com suas reentrâncias, trabéculas, estruturas e orifícios. Maior conhecimento da eletrofisiopatologia da fibrilação atrial, juntamen- te com os avanços tecnológicos, são necessários para que se desenvolvam procedimentos mais seguros e eficazes ${ }^{(57)}$, práticos e reprodutíveis.

NATHAM et al. (58) demonstraram, em indivíduos normais, a presença de tecido atrial estendendo para o interior das veias pulmonares, sendo mais acentuado nas veias pulmonares superiores. Este tecido pode apresentar atividade elétrica e pode ser fonte de taquiarritmias, provavelmente por atividade automática anormal ou atividade deflagrada, gerando extrasístoles isoladas ou salvas de extrasístoles com ciclo curto, em torno de 160 a 260 ms., as quais podem provocar FA.

Ao originar uma arritmia, no interior das veias pulmonares, as deflexões de duplo potencial se invertem e aparece a deflexão em espícula, demonstrando que a ativação elétrica tem origem nas fibras musculares, no interior das veias pulmonares. Não se conhece, ainda, porque o tecido atrial, no interior das veias pulmonares, se torna arritmogênico segundo trabalhos de HAISSAGUERRE et al. $(59,60)$.

A dificuldade em mapear e realizar a ablação dos focos ectópicos das veias pulmonares fez com que se desenvolvesse novas técnicas de ablação, com abordagem anatômica.

LESH et al.(61) descreveram uma técnica que consiste em fazer lesões que circunscrevem o óstio das veias pulmonares, confinando os estímulos provenientes dos focos ectópicos às veias pulmonares, impedindo que cheguem à musculatura atrial. Utilizaram um transdutor de ultra-som, montado na ponta de um cateter envolvido por um balão. Posiciona-se a ponta do cateter no óstio das veias pulmonares a serem abordadas e infla-se o balão com solução salina. O transdutor de ultra-som emite um feixe perpendicular que se propaga pela solução até chegar à parede do balão, que está em contato com o óstio das veias, produzindo lesões térmicas eletricamente isolantes. Essa técnica é chamada de TTB - USA (Trough the Baloon Ultrasound Ablation).

KALIL et al. (62) mostraram, baseados em evidências de serem as veias pulmonares a origem da maioria da fibrilação atrial e devido à complexidade do procedimento do labirinto, técnica cirúrgica simplificada, realizando incisão única, ao redor dos quatro óstios das veias pulmonares, acrescida de outra, unindo aquela incisão ao anel valvar mitral posterior.

Existe um grande interesse na procura de novas fontes de energia capazes de provocar linhas de bloqueio permanente, tais como: termocautério (bisturi elétrico), radiofreqüência, laser, microondas (63) e ultra-som (64). 
A corrente contínua, através do bisturi elétrico, não nos parece adequada, pois não provoca lesões uniformes da parede atrial, com risco de rotura e carbonização, com probabilidade de formação de êmbolos.

A forma de energia mais utilizada para ablação com cateter é a radiofreqüência, que é uma corrente alternada, liberada na forma de onda sinusoidal contínua, não modulada, que é também utilizada para realizar linhas de ablação endocárdica durante procedimento cirúrgico.

HINDRIKS et al. (65), em 1997 e HINDRIKS et al. (66), em 1999, mostraram experiência, com uso de ablação com radiofreqüência, durante procedimento cirúrgico para tratamento de doença valvar. Do mesmo modo, MELO et al. (67-69), em Portugal, mostraram resultados favoráveis, com uso da radiofreqüência intra-operatória para tratamento da fibrilação atrial.

BENUSSI et al. (70) utilizaram a radiofreqüência por via epicárdica nas veias pulmonares durante procedimento cirúrgico.

As lesões miocárdicas da radiofreqüência são produzidas pelo aquecimento resistivo do tecido. Uma grande limitação do sucesso, a longo prazo, da ablação por cateter para o "flutter" atípico e fibrilação atrial, parece ser a dificuldade de criar linhas ininterruptas de bloqueio da transmissão elétrica. Além disso, o aumento constante da impedância, pela carbonização da ponta do cateter, obriga a interrupção da ablação para sua limpeza, já que se trabalha sob visão direta, sem sangue, diferentemente da ablação endocárdica.

Iniciou-se a casuística com a utilização da radiofreqüência, para realização das linhas de ablação intra-operatória ${ }^{(71)}$, com cateter de ablação, tendo sido operados 3 pacientes, com reversão ao ritmo sinusal.

Considerando a experiência no uso do bisturi de ultra-som (UltraCision ${ }^{\circledR}$ ) nas cirurgias para tratamento de taquicardia ventricular refratária (72), foi iniciado o uso do bisturi harmônico, que é bastante utilizado em cirurgias videolaparoscópicas, como descrito por HAMBLEY et al. (73) e AMARAL et al. (74-77), para realização das linhas de ablação intra-operatórias no átrio direito e esquerdo.

O uso do ultra-som, além de facilitar o procedimento, produz lesões mais homogêneas e uniformes, criando necrose transmural, provocando, aparentemente, linhas de bloqueio permanente.

Com base nestes estudos e com os melhores conhecimentos da eletrofitopatogênese da fibrilação atrial, desenvolveu-se a presente técnica cirúrgica alternativa, com menor tempo de execução, para os pacientes com fibrilação atrial crônica e portadores de doença valvar ou congênita, que tenham indicação para tratamento operatório.
A realização da compartimentação atrial, pela ablação transoperatória com bisturi de ultra-som, além de facilitar e diminuir o tempo operatório, permitiu um maior entendimento do papel do átrio esquerdo na fibrilação crônica - como a grande maioria dos pacientes reverteu ao ritmo sinusal, logo após a realização somente das linhas do átrio esquerdo, supõe-se que a região das veias pulmonares e os tecidos ao seu redor desempenham papel importante no desencadeamento e manutenção da fibrilação atrial crônica. $O$ restante do átrio esquerdo e o átrio direito aparentam ter papel passivo, pois o isolamento da área posterior do átrio esquerdo, aonde estão os orifícios das veias pulmonares, foi capaz de interromper a maioria das fibrilações atriais, conforme descrito por KOTTKAMP et al. ${ }^{(78)}$.

Todavia, visando garantir o resultado e baseado em trabalhos de JASBICK et al. (14) e BATISTA et al. (16), foram realizadas as manobras complementares: exclusão das aurículas direita e esquerda e redução do átrio esquerdo. Somente em 2 pacientes não se obteve o retorno ao ritmo sinusal e não se tem explicação para o insucesso. Tratava-se de primeira cirurgia e o átrio esquerdo estava pouco aumentado. Em contraste, mesmo em casos de reoperação, com átrio esquerdo grande, houve reversão ao ritmo sinusal. Em um paciente submetido à reoperação, em virtude das aderências no átrio direito, realizouse somente as linhas do átrio esquerdo. Posteriormente se verificou, no pós-operatório tardio, "flutter" atrial atípico, que foi em seguida eliminado, através de ablação por cateter. Passou-se, então, a realizar, sistematicamente, a atriotomia direita, para a confecção das linhas de ablação no átrio direito.

Esta técnica, de fácil execução e baixo índice de complicações, com tempo mínimo de circulação extracorpórea, pode ser executada em todo centro de cirurgia cardíaca, necessitando, somente, do bisturi especializado de ultra-som.

\section{CONCLUSÕES}

O tratamento intra-operatório da fibrilação atrial crônica, com a técnica realizada utilizando ultra-som, possibilitou a reversão imediata para o ritmo sinusal em $\mathbf{8 8 , 8 \%}$ dos pacientes, mantendo-se esse ritmo em $81,4 \%$, por ocasião da alta hospitalar.

A nova abordagem de tratamento intra-operatório da fibrilação atrial crônica, com emprego da técnica de compartimentação atrial, através da confecção de linhas de ablação transcirúrgicas, com bisturi de ultra-som, mostrou-se de simples execução, sem grandes agressões, realizada em curto espaço de tempo e sem complicações relacionadas com o procedimento no per e pós-operatório. 
ABSTRACT: Introduction: The intraoperative atrial compartmentalization was done in 27 patients, utilizing ultrasound energy (US). This source has been more effective in creating deeper and uniform atrial line lesions, without causing carbonization.

Material and Methods: From March 1999 to June 2000, 27 patients (19 females) averaging 36 years old were submitted to this operation. Five were operated for the second time due to mitral valve disorders, 2 for mixomatose valve degenerations, 1 for congenital defect and 1 for lone atrial fibrillation.

Results: The procedure takes 166.6 minutes on average, in each surgery, that is 69.2 minutes for the cardiopulmonary bypass, 39.7 minutes for cardiac arrest by aorta clamping, 12.5 minutes for the right atrial lesion lines and 14 minutes for the left one. The reversion to sinus rhythm was achieved in 24 patients. In 2 the reversion was not obtained and in 1 transient total atrium-ventricular block was observed. No explanation was found for the failure to revert the 2 patients, since both were primarily phase submitted to valve repair operation and the atrium was not very enlarged. In the postoperative phase, atrial fibrillation recurrence was observed in 2 patients and two deaths occurred cuased by pulmonary problem and cardiogenic shock unrelated to the technique. The success rate at the hospital discharge was $81.4 \%$. To all patients were given verapamil or amiodarone for electrical atrium remodeling.

Conclusion: The use of US during mitral valve operation to compartmentalize the atrium, in patients with CAF, makes the reversion to sinus rhythm so easy and fast that is possible to consider employing this technique in patients having CAF associated to other surgical cardiac diseases. The use of US, during cardiac operation, to create the atrial line lesion, modifies and makes easier the Maze procedure, causes less tissue damage, decreases the surgical time and the chance for postoperative complications.

DESCRIPTORS: Atrial fibrillation, surgery. Arrhythmia, therapy. Cardiac surgical procedures, methods.

\section{REFERÊNCIAS BIBLIOGRÁFICAS}

1 Kalman J M \& Tonkin A M - Atrial fibrillation: epidemiologyand the risk and prevention of stroke. Pacing Clin Electrophysiol 1992; 15:1332-8.

2 Gallagher J J, Svenson R H, Kasel J M et al. - Catheter technique for closed-chest ablation of the atrioventricular conduction system. $N$ Engl J Med 1982; 306:194-200.

3 Scheinman M M, Morady F, Hesse D S et al. - catheter induced ablation of the atrioventricular junction to control refractory supraventricular arrhytmias. JAMA 1982; 248: 851-5.

4 Morady $\mathrm{F}$, Calbins $\mathrm{H}$, Langberf $\mathrm{J} \mathrm{J} \mathrm{et} \mathrm{al.} \mathrm{-} \mathrm{A} \mathrm{prospective}$ randomized comparison of direct current and radiofrequency ablation of the atrioventricular junction. J Am Coll Cardiol 1993; 21:102-9.

5 Olgin J E \& Sheinman M M - Comparison of high energy direct current and radiofrequency catheter ablation of the atrioventricular junction. J Am Coll Cardiol 1983; 21: 557-64.

6 Willians J M, Ungerleider R M, Zofland G K, Cox J L Left atrial isolation. J Thorac Cardiovasc Surg 1980; 80: 373-80.

7 Graffigna A, Pagani F, Menzioni G, Salerno J, Vigano M - Left atrial isolation associated with mitral valve operation. Ann Thorac Surg 1992; 54: 1093-8.
8 Guiradon G M, Campbel C S, Jones D L et al. - Combined sino atrial node atrioventricular node isolation: a surgical alternative to his bundle ablation in patients with atrial fibrillation. Circulation 1985; 72 (suppl. 3): 220. (Abstract)

9 Cox J L - The surgical treatment of atrial fibrillation: IVsurgical technique. J Thorac Cardiovasc Surg 1991; 101: 584-92.

10 Cox J L, Boineau J P, Schuessler R B, Jaquiss R D, Lappas D G - Modification of the maze procedure for atrial flutter and atrial fibrillation: I- rationale and surgical results. J. Thorac Cardiovasc Surg 1995; 110: 473-94.

11 Cox J L, Jaquiss R D, Schuessler R B, Boineau J P Modification of the maze procedure for atrial flutter and atrial fibrillation: II- surgical technique of the maze III procedure. J Thorac Surg 1995; 110: 485-95.

12 Jatene A D, Sosa E, Tarasoutchi F, Jatene M B, Pomerantzef P M A - Tratamento cirúrgico da fibrilação atrial. "procedimento do labirinto": experiência inicial. Rev Bras Cir Cardiovasc 1992; 7: 107-11.

13 Jatene M B, Sosa E, Jatene F B et al. - Evolução tardia da operação de cox para fibrilação atrial em valvopatia mitral. Rev Bras Cir Cardiovasc 1995; 10: 18-24.

14 Jazbick J C, Coutinho J H, Amar M R et al. - Tratamento cirúrgico da fibrilação atrial em pacientes com insuficiência mitral: proposta inicial de uma nova abordagem cirúrgica. Rev SOCERJ 1993; 6: 142-5. 
Brick A V, Seixas T, Portilho C, Peres A K, Vieira Jr J J, Melo Neto R, Araújo J M - Tratamento intra-operatório da fibrilação atrial crônica com ultra-som. Rev Bras Cir Cardiovasc 2001; 16(4): 337-49.

Gregori Jr. F, Cordeiro, Goulart M A et al. - Técnica de Cox sem crioablação para tratamento cirúrgico da fibrilação atrial. Rev Bras Cir Cardiovasc 1993; 8: 220-4.

16 Batista R J V, Franzoni M, Precoma D V et al. Autotransplante cardíaco: um novo método no tratamento de problemas cardíacos complexos. Rev Bras Cir Cardiov 1995; 10: 90-100.

17 Grimberg M, Solimene M C, Belotti G et al. - Estenose mitral calcificada: aspectos clínicos e cirúrgicos. Arq Bras Cardiol 1975; 28: 17-24.

18 Maratia C, Kalil R A K, Santanna et al. - Fatores preditivos de reversão a ritmo sinusal após intervenção na valva mitral em pacientes com fibrilação atrial crônica. Rev Bras Cir Cardiovasc 1997; 12: 17-23.

19 Cox J L, Schuessler R B, D'Agostino Jr H J et al. - The surgical treatment of atrial fibrillation: III- development of definitive surgical procedure. J Thorac Cardiovasc Surg 1991; 101: 569-83.

20 Rodrigues T R \& Miranda R C - Aspectos epidemiológicos, etiologia e classificação da fibrilação atrial. Replamba 1999; 12: 165-70.

21 Kannel W B - Epidemiologic features of atrial fibrillation: the Framinghan study. N Engl J Med 1982; 306: 1018-22.

22 Middlekauf H R, Stevenson W G, Stevenson L W Prognostic significance of atrial fibrillation in advanced heart failure: a study of 390 patients. Circulation 1991; 84: 40-8.

de Paola A A V \& Veloso H H - Efficacy and safety of sotalol versus quinidine for the maintenance of synus rhythm after conversion of atrial fibrillation. $A m \mathrm{~J}$ Cardiol 1999; 84: 1033-7.

24 de Paola A A V, Veloso $\mathrm{H} \mathrm{H} \mathrm{H}$, Figueiredo et al. Fibrilação atrial. Rev Soc Cardiol Estado de São Paulo 1988; 1: 46-56.

Moraes C R, Buffolo E, Lima R et al. - Surgical treatment of endomyocardial fibrosis. $J$ Thorac Cardiovasc Surg 1983; 85: 738-45.

Chua Y I, Schaff H V, Orszulak T A, Morris J J - Outcome of mitral valve repair in patients with preoperative atrial fibrillation: should the maze procedure be combined with mitral valvuloplasty? J Thorac Cardiovasc Surg 1994; 107: 408-15.

27 Wipf J E \& Lipsky B A - Atrial fibrillation: thomboembolic risk and indications for anticoagulation. Arch Intern Med 1990; 150: 1598-603.

28 Fisher C M - Embolism in atrial fibrillation. In: Kulbertus H E, Olsson S B, Schlepper M, eds. Atrial Fibrillation. Sweden: A.B. Hassie, 1982. p. 192-210.

29 Moe G K - On the multiple wavelet hypothesis of atrial fibrillation. Arch Int Pharmacodyn Ther 1962; 140: 182-3.
30 Allessie M A, Lammers W J E P, Bonkefin et al. - Experimental evaluation of moe's multiple wavelet hipothesis of atrial fibrillation. In: Zipes D P, Jalife J, ed. Cardiac electrophysiology and arrhythmias. New York: Frune \& Stratton,1985. p.256.

31 Fenelon G \& de Paula A - Novas idéias sobre os mecanismos eletrofisiológicos da fibrilação atrial e suas implicações terapêuticas. Replamba 2000; 13: 5-12.

32 Kannel W B, Wolf $\mathrm{P}$ A, Bem J A, Min E J et al. Prevalence, incidence, prognosis and predisponing conditions for atrial fibrillation: population based estimates. Am J Cardiol 1988; 82: 2N-9.

Moe G K \& Abikskov J A - Atrial fibrillation as a self sustaning arrhytmia independent of local discharge. Am Heart J 1959; 58: 59-70.

34 Rensma P, Alessie M A, Lammers W J, Bonke F I, Schalij M J - Lengh of excitation wave and susceptibility to reentrant atrial arrhytmias in normal conscious dog. Circ Res 1988; 62: 395-410.

35 Falk R - Etiology and complications of atrial fibrillation: insights from pathology studies. Am J Cardiol 1988; 82: $10 \mathrm{~N}-17$.

36 Lid D, Fareh S, Leung T K, Nattel S - Promotion of atrial fibrillation by heart failure in dogs: atrial remodeling of a different sort. Circulation 1999; 100: 87-95.

37 Botteron G W \& Smith J M - Quantitative assessment of the spatial organization of atrial fibrillation in the intact human heart. Circulation 1996; 93: 513-8.

38 Cox J L, Canavan T E, Schuessler R B et al. - The surgical treatment of atrial fibrillation: II- intraoperative electrophysiologic mapping and description of the electrophysiologic basis of atrial flutter and atrial fibrillation. J Thorac Cardiovasc Surg 1991; 101: 406-26.

39 Cox J L, SchuesSler R B, Boineau J P - The surgical treatment of atrial fibrillation: I- summary of the current concepts of the mechanism of atrial flutter and atrial fibrillation. J Thorac Cardiovasc Surg 1991; 101: 402-5.

40 Cox J L, Boineau J P, Schuessler R B et al. - Operations for atrial fibrillation. Clin Cardiol 1991; 14: 827-34.

41 Cox J L, Boineau J P, Schuessler R B, Kater K M, Lappas D G - Five year experience with the maze procedure for atrial fibrillation. Ann Thorac Surg 1993; 56: $814-24$

42 Jatene M B, Barbero-Marcial M, Tarasoutchi F, Cardoso R A, Pomerantzef P M A, Jatene A D - Influência da operação de Cox no tratamento da fibrilação atrial em valvopatia reumática: análise comparativa de resultados imediatos e tardios. Rev Bras Cir Cardiovasc 1988; 13: 105-19.

43 Jatene $M$ - Cirurgia do labirinto: técnica e resultados. Replamba 2000: 13: 13-8.

44 Sandoval N, Velasco V M, Orjuela H et al. - Concomitant mitral valve or atrial septal defect surgery and the modified Cox - maze procedure. Am J Cardiol 1996; 77: 591-6. 
Brick A V, Seixas T, Portilho C, Peres A K, Vieira Jr J J, Melo Neto R, Araújo J M - Tratamento intra-operatório da fibrilação atrial crônica com ultra-som. Rev Bras Cir Cardiovasc 2001; 16(4): 337-49.

McCarthy P M, Castle L W, Maloney J D et al. - Initial experience with the maze procedure for atrial fibrillation. J Thorac Cardiovasc Surg 1993; 105: 1077-87.

Kosakai Y, Kawaguchi A T, Isolve F et al. - Cox maze procedure for chronic atrial fibrillation associated with mitral valve disease. J Thorac Cardiovasc Surg 1994; 108: 1049-55.

47 Kawaguchi A T, Kosakai Y, Sasako Y, Eishi K, Nakano $\mathrm{K}$, Kawashima $\mathrm{Y}$ - Risks and benefits of combined maze procedure for atrial fibrillation associated with organic heart disease. J Ann Coll Cardiol 1996; 28: 985-90.

Losano F L - Técnica de maze para el tratamiento de la fibrilação auricular. experiência inicial. Rev Esp Cardiol 1998; 51: 901-7.

Avitall B, Hare J, Mughal $\mathrm{K}$ et al. - Ablation of atrial fibrillation in a dog model J Am Coll Cardiol 1994; 482: 276 A. (Abstract)

50 Elvan A, Pride H P, Eble J N, Zipies D P - Radiofrequency catheter ablation of the atrial reduces inducibility an duration of fibrillation in dogs. Circulation 1994; 90: 335.

51 Haissaguerre M, Gencell L, Fischer B et al. - Successful catheter ablation of atrial fibrillation. J Cardiovasc Electrophysiol 1994; 5: 1045-52.

52 Haissaguerre M, Jais P, Shah D C et al. - Right and left atrial radiofrequency catheter therapy of paroxysmal atrial fibrillation. J Cardiovasc Electrophysiol 1996; 7: 1132-44.

53 Swartz J F, Pellerseis G, Silvers J, Patten L, Cervantez D - A catheter-based curative approach to atrial fibrillation in humans. Circulation 1994; 90: I- 335.

Robbins I M, Colvin E V, Doyle T P et al. - Pulmonary vein stenosis after catheter ablation of atrial fibrillation. Circulation 1998; 98: 1769-75.

Gaita F, Ricardi R, Calo L et al. - Atrial mapping and radiofrequency catheter ablation in patients with idiopathic atrial fibrillation: electrophysiological findings and ablation results. Circulation 1998; 97: 2136-45.

56 Garg A, Finneran W, Mollerus M et al. - Right atrial compartimentalization using radiofrequency catheter ablation for management of patients with refractory atrial fibrillation. J Cardiovasc Electrophysiol 1999; 10: $763-71$

57 Jorge J C M, Nadalin E, Silva M A - Procedimentos ablativos por cateter no tratamento da fibrilação atrial. Replamba 2000; 13: 19-29.

58 Natham M \& Eliakin M - The junction between the left atrium and the pulmonary veins: an anatomic study of human heart. Circulation 1966; 34: 412-22.
59 Haissaguerre M, Jais P, Shas D C et al. - Spontaneous initiation of atrial fibrillation by ectopic beats originating in pulmonary veins. N Engl J Med 1988; 399: 659-66.

60 Haissaguerre $\mathrm{M}$, Jais $\mathrm{P}$, Shas D C et al. - End point a successful ablation of atrial fibrillation initiated from the pulmonary veins. Pace 1999; 22: 711.

61 Lesh M D, Diederich C, Guerra P G, Goseki Y, Sparks $P B-A n$ anatomic approach to prevention of atrial fibrillation: pulmonary vein isolation whit though The balloon ultrasound ablation (TTB - USA) Thorac Cardiovasc Surg 1999; 47 (suppl 3): 347-51.

62 Kalil R A K, Lima G G, Abrahão R et al. - Técnica cirúrgica simplificada pode ser eficaz no tratamento da fibrilação atrial crônica secundária à lesão valvar mitral? Rev Bras Cir Cardiovasc 2000; 15:129-35

63 Krum D, Shetty S, Mare J et al. - Characteristics of myocardial lesions created using microwave energy: optimization of antena design. Circulation 1994; 90 : 1271. (Abstract)

64 He D S, Simmons W N, Zimmer et al. - Comparison of several ultrasonic frequencies for cardiac ablation. Circulation 1994; 90: I- 271. (Abstract)

65 Hindricks G, Kottkamp H, Hammel D et al. - Radiofrequency ablation of atrial fibrillation: early clinical experiency with a catheter-guided intraoperative aproach. In: XIXth Congress of the European Society of Cardiology - Stockholm, Sweden. August 24-28, 1997. p.893.

66 Hindricks F, Mohr W, Autschbach R, Kottkamp H Antiarrhytmic surgery for treatment of atrial fibrillation: new concepts. Thorac Cardiovasc Surg 1999, 47 (suppl 3): 365-9.

67 Melo J Q, Neves J, Adragao P et al. - When and how to report results of surgery on atrial fibrillation. Eur J Cardiothorac Surg 1997; 12: 739-45.

68 Melo J Q, Neves J, Adragao P et al. - Cirurgia da fibrilação auricular utilizando radiofreqüência intra-operatória. Rev Port Cardiol 1988; 17: 377-9.

69 Melo J Q, Adragao P, Neves J et al. - Surgery for atrial fibrillation using radiofrequency catheter ablation: assessment of results at one year. Eur $J$ Cardiothoracic Surg 1999; 15: 851-5.

70 Benussi S, Pappone C, Nascimbene S et al. - A simple way to treat chronic atrial fibrillation during mitral valve surgery: the epicardial radiofrequency approach. Eur J Cardiothoracic Surg 2000; 17: 524-9.

71 Brick A V, Seixas S T, Peres S A et al. - Reversão da fibrilação atrial crônica pela técnica do labirinto com aplicação de radiofreqüência e ultra-som transoperatório. Rev Bras Cir Cardiovasc 1999; 14: 290-7. 
Brick A V, Seixas T, Portilho C, Peres A K, Vieira Jr J J, Melo Neto R, Araújo J M - Tratamento intra-operatório da fibrilação atrial crônica com ultra-som. Rev Bras Cir Cardiovasc 2001; 16(4): 337-49.

72 Brick A V, Seixas T N, Portilho C F, Vieira Jr. J J, Mattos J V, Peres A K - Tratamento cirúrgico da taquicardia ventricular refratária: nova proposta técnica. Rev Bras Cir Cardiovasc 1997; 12: 269-73.

73 Hambley R, Hebda P A, Abell E, Cohen B A, Jegasothy $B \vee$ - Wound healing of skin incisions procuded by ultrassonically vibration knife, scalpel, electrosurgery and carbon dioxide laser. J Dermatol Surg Oncol 1988; 14: 1213-7.

74 Amaral J F - Laparoscopic application of a ultrassonically activated scalpel. G I Endoscopy Clinics North Am 1993; 3: 381-91.

75 Amaral J F \& Chrostek C - Comparison of the ultrassonically activated scalpel to electrosurgery and laser surgery for laparoscopic surgery. Proceedings of the Society of American Gastrointestinal Endoscopic Surgeons. Phoenix: April, 1993.

76 Amaral J F - The experimental development of an ultrasonically activated scalpel for laparoscopic use. Surg Laparosc Endosc 1994; 4: 92-9.

77 Amaral J F - Ultrasonic dissection. Endosc Surgery \& Allied Technology 1994; 2: 181-5.

78 Kottkamp H, Hindricks G, Hammel D et al. - Intraoperative radiofrequency ablation of chronic atrial fibrillation: a left atrial curative approach by elimination of anatomic "anchor" reentrant circuits. J Cardiovasc Electrophysiol 1999; 10: 772-80. 